\title{
Microwave reflectometry for the study of density fluctuations in tokamak plasmas
}

\author{
E. Mazzucato and R. Nazikian \\ Princeton Plasma Physics Laboratory \\ Princeton University \\ Princeton, N. J. 08543
}

\begin{abstract}
The effect of small scale density fluctuations on the propagation of electromagnetic waves in an inhomogeneous magnetized plasma in the presence of a cutoff is investigated. It is shown that, provided the fluctuation scale length is greater than the free space wavelength of an incident plane wave, the scattered field is strongly enhanced from fluctuations near the turning point. Numerical results for wave propagation in a tokamak plasma demonstrate the feasibility of reflectometry for the localized measurement of density fluctuations in the range $k_{\perp} \rho_{i} \ll 1$.
\end{abstract}




\section{Introduction}

The causes of anomalous transport in tokamak plasmas are still unknown. A popular conjecture, which finds limited support in theory and experiments, is that the thermal and particle transport is enhanced by the existence of fine scale turbulence. Indeed, theory predicts a large variety of plasma waves which are driven unstable by density and temperature gradients, by dissipative effects and by magnetically trapped particles (Kadomtsev and Pogutse, 1971; Tang, 1978; Horton, 1984). Experimentally, observations with microwave (Mazzucato, 1976) and laser (Surko and Slusher, 1976) scattering have revealed the existence of a small scale turbulence in rough agreement with theoretical predictions.

Present observations show that the level of density fluctuation $\tilde{n}$ increases as the perpendicular wave number $k_{\perp}$ decreases, as predicted by the mixing length criterion $\tilde{n} / n \approx 1 / k_{\perp} L_{n}$ [where $L_{n}=(d \ln n / d r)^{-1}$ is the density scale length]. This makes the interpretation of experimental data very difficult as most scattering techniques detect long wavelength fluctuations with only poor spatial resolution. On the other hand, very long wavelength modes, i.e., modes with small poloidal and toroidal mode numbers, do not show correspondingly large fluctuation levels. This implies that the turbulence spectrum must turn over at some value of $k_{\perp}$ where present observations lack spatial resolution. This is of considerable importance since turbulent fluctuations with amplitudes below the mixing length level and wavelengths much longer than the ion Larmor radius $\rho_{i}$ could theoretically account for the observed plasma transport in tokamak (Liewer, 1985; Haas and Thyagaraja, 1986). Clearly the measurement of long wavelength fluctuations with improved spatial resolution over existing scattering techniques is needed.

One technique with the potential for providing spatially localized measurements of long wavelength density fluctuations is microwave reflectometry. In fact, the first experimental evidence for the existence of a fine scale turbulence in tokamaks was obtained using microwave reflectometry on the adiabatic toroidal 
compressor (ATC) tokamak (Mazzucato, 1975). This method, widely employed in atmospheric studies, measures the reflection of electromagnetic waves from the plasma cutoff to obtain the electron density profile in inhomogeneous plasmas. The system can be considered a special kind of interferometer where the phase of the received wave is determined by the refractive index along the wave trajectory and also by changes in the position of the reflecting layer.

Enhanced scattering from fluctuations at the cutoff in isotropic plasmas was first addressed by Pitteway (1958) in reference to radio wave propagation in the ionosphere. In this paper, we address the issue of wave propagation in anisotropic plasmas and assess the relative capabilities of $\mathrm{O}$-mode and X-mode reflectometry for the local measurement of density fluctuations in tokamaks.

The paper is organized as follows: the basic equations for the scattered field in the Born approximation are derived in $\S 2$ for both the ordinary and the extraordinary mode of propagation. Some numerical examples are presented in $\S 3$ which simulate the case of a large tokamak plasma. Finaily, our conclusions are presented in $\S 4$.

\section{Basic Equations}

In this section, we derive the equations for the electromagnetic field scattered by small density fluctuations in a magnetized plasma.

A plane electromagnetic wave $\mathbf{E}=\mathbf{E}_{0} \exp i\left(\omega_{0} t-\mathbf{k}_{0} \cdot \mathbf{r}\right)$ is launched into a plasma with a magnetic field B which, in Cartesian orthogonal coordinates $\left(x^{\prime}, y^{\prime}, z^{\prime}\right)$, is aligned in the $y^{\prime}$ direction and is only a function of $z^{\prime}$. The plasma occupies the region $z^{\prime}>0$ with the electron density distribution given by

$$
n_{e}=\bar{n}_{e}\left(z^{\prime}\right)+\tilde{n}_{e}\left(x^{\prime}, z^{\prime}, t\right)
$$

i.e., where a plane stratified plasma equilibrium is perturbed by weak $\left(\left|\tilde{n}_{e}\right|<<\right.$ $\bar{n}_{e}$ ) irregularities which are uniform along magnetic field lines. While we assume that the free space wavelength $\lambda_{0}$ is comparable to the spatial scale length $\Lambda$ of 
plasma fluctuations, we assume the wave frequency $\omega_{0}$ to be much greater than the bandwidth of density fluctuations so that the time dependence of Eq. (1) can be ignored. Finally, we assume $\mathbf{k}_{0} \cdot \mathbf{B}=0$. This geometry reproduces the typical reflectometer configuration used in the investigation of density fluctuations in tokamaks (Mazzucato, 1975; Cripwell et al., 1989), the major simplification being the omission of the magnetic shear in the description of the ware propagation which is justified by its smallness in this type of magnetic configuration.

Under these assumptions, the electromagnetic field may be separated into two independent modes of propagation, each described by a scalar differential equation in the two variables $x^{\prime}$ and $z^{\prime}$ (Budden, 1961). The dependent variable is the $y^{\prime}$ component of the electric field $\mathrm{E}$ for the ordinary wave, and the $y^{\prime}$ component of the magnetic field $\mathrm{H}$ for the extraordinary wave. Using standard notations and introducing the change of coordinates $(x, y, z)=\left(k_{0} x^{\prime}, k_{0} y^{\prime}, k_{0} z^{\prime}\right)$, the equation for $E$ is

$$
\nabla^{2} \mathrm{E}+\epsilon_{0} \mathrm{E}=0
$$

while the equation for $\mathrm{H}$ is

$$
\nabla^{2} \mathrm{H}-\frac{1}{\epsilon_{1}} \nabla \mathrm{H} \cdot \nabla \epsilon_{1}+i \frac{\epsilon_{1}}{\epsilon_{2}^{2}} \mathbf{i}_{y} \cdot \nabla \mathrm{H} \times \nabla \epsilon_{2}+\epsilon_{1} \mathrm{H}=0
$$

where $\mathbf{i}_{y}$ is the unit vector along the $y$-axis, and

$$
\epsilon_{0}=1-\frac{X}{U}, \epsilon_{1}=\frac{(U-X)^{2}-Y^{2}}{U(U-X)-Y^{2}}, \epsilon_{2}=\frac{(U-X)^{2}-Y^{2}}{X},
$$

with $\mathrm{X}=\omega_{p}^{2} / \omega^{2}, \omega_{p}^{2}=4 \pi n_{e} e^{2} / m_{e}, Y=\omega_{c} / \omega, \omega_{c}=e|\mathbf{B}| / m_{e} c, U=1-i \nu / \omega$, and $\nu<<\omega$ a small effective collision frequency which takes into account weak wave damping. When $\nu=0, \epsilon_{0}=0$ for $\mathrm{X}=1$ and $\epsilon_{1}=\epsilon_{2}=0$ for $\mathrm{X}=1 \pm \mathrm{Y}$.

Using Eq. (1) we may put Eq. (4) in the form

$$
\epsilon_{i}=\bar{\epsilon}_{i}(z)+\tilde{\epsilon}_{i}(x, z), \quad i=0,1,2
$$


where $\left|\widetilde{\epsilon}_{i}\right| \ll<1$. We shall assume that $d \bar{\epsilon}_{i}(z) / d z \neq 0$ at $z=z_{c}$, and that the thickness of the evanescent region behind the cutoff is many free space wavelengths such that tunnelling effects may be ignored. Then, we may proceed by making the Ansatz, to be verified a posteriori, that

$$
\mathrm{E}=\mathrm{E}_{0}+\sum_{n>0} \mathrm{E}_{n}
$$

where $\left|E_{0}\right| \sim O(1)$ and $\left|E_{n>0}\right| \sim O\left(\tilde{\epsilon}_{0}^{n}\right)$. From the two lowest order terms of Eq. (2) we obtain for the ordinary mode

$$
\nabla^{2} \mathrm{E}_{0}+\bar{\epsilon}_{0}(z) \mathrm{E}_{0}=0
$$

and

$$
\nabla^{2} \mathrm{E}_{1}+\bar{\epsilon}_{0}(z) \mathrm{E}_{1}=-\widetilde{\epsilon}_{0}(x, z) \mathrm{E}_{0}(z) .
$$

Similarly, by assuming for the extraordinary mode

$$
\mathrm{H}=\mathrm{H}_{0}+\sum_{n>0} \mathrm{H}_{n}
$$

with $\left|\mathrm{H}_{0}\right| \sim O(1)$ and $\left|\mathrm{H}_{n>0}\right| \sim O\left(\widetilde{\epsilon}_{1}^{n}\right)$, we obtain

$$
\nabla^{2} \mathrm{H}_{0}-\frac{1}{\bar{\epsilon}_{1}} \nabla \mathrm{H}_{0} \cdot \nabla \bar{\epsilon}_{1}+i \frac{\bar{\epsilon}_{1}}{\bar{\epsilon}_{2}^{2}} \mathbf{i}_{y} \cdot \nabla \mathrm{H}_{0} \times \nabla \bar{\epsilon}_{2}+\bar{\epsilon}_{1} \mathrm{H}_{0}=0
$$

and

$$
\begin{aligned}
& \nabla^{2} \mathrm{H}_{1}-\frac{1}{\bar{\epsilon}_{1}} \nabla \mathrm{H}_{1} \cdot \nabla \bar{\epsilon}_{1}+i \frac{\bar{\epsilon}_{1}}{\bar{\epsilon}_{2}^{2}} \mathbf{i}_{y} \cdot \nabla \mathrm{H}_{1} \times \nabla \bar{\epsilon}_{2}+\bar{\epsilon}_{1} \mathrm{H}_{1}= \\
& \nabla \mathrm{H}_{0} \cdot \nabla \frac{\tilde{\epsilon}_{1}}{\bar{\epsilon}_{1}}-i \frac{\bar{\epsilon}_{1}}{\bar{\epsilon}_{2}} \mathbf{i}_{y} \cdot \nabla \mathrm{H}_{0} \times\left[\nabla \frac{\tilde{\epsilon}_{2}}{\bar{\epsilon}_{2}}+\frac{1}{\bar{\epsilon}_{2}}\left(\frac{\widetilde{\epsilon}_{0}}{\bar{\epsilon}_{0}}-\frac{\tilde{\epsilon}_{1}}{\bar{\epsilon}_{1}}\right) \nabla^{2} \bar{\epsilon}_{2}\right]-\tilde{\epsilon}_{1} \mathrm{H}_{0} .
\end{aligned}
$$

Let us first consider the case of the ordinary mode with an incident plane wave which in vacuum takes the form $\exp \left[i\left(\omega_{0} t-\alpha_{0} x-\beta_{0} z\right)\right]$, with $\beta_{0}=(1-$ $\left.\alpha_{0}^{2}\right)^{1 / 2}$ so as to satisfy the wave equation for free space propagation. Taking solutions of Eq. (6) in the form

$$
E_{0}=E_{0}(z) \exp \left(-i \alpha_{0} x\right)
$$


we obtain

$$
\frac{d^{2} E_{0}}{d z^{2}}+\left[\bar{\epsilon}_{0}(z)-\alpha_{0}^{2}\right] E_{0}=0
$$

Assuming a linear density distribution near the turning point and introducing the change of variable $\zeta=\left|d \bar{\epsilon}_{0} / d z\right|^{1 / 3}\left(z_{0}-z\right)$, with $\bar{\epsilon}_{0}\left(z_{0}\right)=\alpha_{0}^{2}$, we may write Eq. (10) as (Ginzburg, 1961)

$$
\frac{d^{2} E_{0}}{d \zeta^{2}}+\zeta E_{0}=0
$$

Two independent solutions are the Airy functions $A i(\zeta)$ and $B i(\zeta)$ with asymptotic forms

$$
\begin{aligned}
& A i(\zeta) \approx \frac{1}{\sqrt{\pi} \zeta^{1 / 4}} \sin \left(\frac{2}{3} \zeta^{3 / 2}+\frac{\pi}{4}\right) \\
& B i(\zeta) \approx \frac{1}{\sqrt{\pi} \zeta^{1 / 4}} \cos \left(\frac{2}{3} \zeta^{3 / 2}+\frac{\pi}{4}\right)
\end{aligned}
$$

for $\zeta>>1$, and

$$
\begin{aligned}
& A i(\zeta) \approx \frac{1}{2 \sqrt{\pi}(-\zeta)^{1 / 4}} \exp \left(-\frac{2}{3}(-\zeta)^{3 / 2}\right) \\
& B i(\zeta) \approx \frac{1}{2 \sqrt{\pi}(-\zeta)^{1 / 4}} \exp \left(\frac{2}{3}(-\zeta)^{3 / 2}\right)
\end{aligned}
$$

for $\zeta<<-1$. Since

$$
\frac{2}{3} \zeta^{3 / 2}=\frac{2}{3}\left|\frac{d \bar{\epsilon}_{0}}{d z}\right|_{z=z_{0}}^{1 / 2}\left(z_{0}-z\right)^{3 / 2} \approx \int_{z}^{z_{0}}\left(\epsilon_{0}\left(z^{\prime}\right)-\alpha_{0}^{2}\right)^{1 / 2} d z^{\prime},
$$

the asymptotic expansions tend towards the approximation of geometrical optics. In the vacuum region this gives

$$
E_{0}(z)=\exp \left(-i \beta_{0} z\right)+\exp \left[i\left(\beta_{0} z+2 \int_{0}^{z_{0}}\left(\epsilon_{0}\left(z^{\prime}\right)-\alpha_{0}^{2}\right)^{1 / 2} d z^{\prime}+\frac{\pi}{2}\right)\right] .
$$

This expression forms the basis for reflectometry measurements of the average density distribution in nonuniform plasmas (Doane et al., 1981).

We now consider perturbations of the type

$$
\widetilde{\epsilon}_{0}(x, z)=\widetilde{\varepsilon}_{0}(z) \exp (-i p x)
$$


and solutions of Eq. (7) of the form

$$
\mathrm{E}_{1}=E_{1}(z) \exp \left(-i \alpha_{1} x\right)
$$

where $\alpha_{1}=\alpha_{0}+p$. The equation for the scattered field is then

$$
\frac{d^{2} E_{1}}{d z^{2}}+\left[\bar{\epsilon}_{0}(z)-\alpha_{1}^{2}\right] E_{1}=-\widetilde{\varepsilon}_{0}(z) E_{0}(z)
$$

which may be solved using the method of Variation of Parameters (Jeffreys and Jeffreys, 1972). Let $z=z_{1}$ be the point where $\epsilon_{0}\left(z_{1}\right)=\alpha_{1}^{2}$, and functions $A(z)$, $B(z)$ are two independent solutions of the corresponding homogeneous equation having asymptotic expansions similar to Eqs. (12) and (13) with $z_{0}$ replaced by $z_{1}$ and $\alpha_{0}$ replaced by $\alpha_{1}$. Near the turning point $z=z_{1}$, these functions are approximated by the Airy functions, while away from the turning point the independent solutions are given by the WKB approximations (Heading, 1962). Alternatively, the solutions of the homogeneous equation may be evaluated numerically.

According to the method of Variation of Parameters, the solution of Eq. (15) may be cast in the form

$$
E_{1}(z)=-\int_{-\infty}^{z} \widetilde{\varepsilon}_{0}(v) \frac{y_{1}(v) y_{2}(z)}{W} E_{0}(v) d v-\int_{z}^{\infty} \widetilde{\varepsilon}_{0}(v) \frac{y_{1}(z) y_{2}(v)}{W} E_{0}(v) d v
$$

where the functions $y_{1}(z)$ and $y_{2}(z)$ are two independent solutions of the homogeneous equation, and $W$ is the Wronskian which in this case is a constant. The functions $y_{1}$ and $y_{2}$ are expressible as linear combinations of $A(z)$ and $B(z)$ which satisfy the radiation conditions at $z= \pm \infty$. Thus, since $E_{1}(z) \rightarrow 0$ as $z \rightarrow \infty$, then $y_{2}(z)=A(z)$, and since $E_{1}(z) \rightarrow \exp \left[i\left(1-\alpha_{1}^{2}\right)^{1 / 2} z\right]$ as $z \rightarrow-\infty$, then $y_{1}(z)=B(z)+i . A(z)$ so the solution of Eq. (15) takes the form

$$
\begin{aligned}
E_{1}(z) & =-\left[\frac{A(z)}{W} \int_{-\infty}^{z} \widetilde{\varepsilon}_{0}(v)[B(v)+i . A(v)] E_{0}(v) d v\right. \\
& \left.+\frac{[B(z)+i . A(z)]}{W} \int_{z}^{\infty} \widetilde{\varepsilon}_{0}(v) A(v) E_{0}(v) d v\right] .
\end{aligned}
$$


In the vacuum region, apart from a constant phase, we obtain

$$
E_{1}(z)=\beta_{1}^{-1} \exp \left(i \beta_{1} z\right) \int_{0}^{\infty} \tilde{\varepsilon}_{0}(v) G_{0}(v) d v
$$

with

$$
G_{0}(v)=-A(v) E_{0}(v)
$$

where $\beta_{1}=\left(1-\alpha_{1}^{2}\right)^{1 / 2}$, and the functions $A(z)$ and $B(z)$ are normalized to a unit amplitude in the vacuum region. Equation (17) agrees with the result of Pitteway (1958) derived using an alternative approach.

The case of the extraordinary mode may be solved using a similar procedure. Again, given an incident plane wave of the form $\exp \left[i\left(\omega t-\alpha_{0} x-\beta_{0} z\right)\right]$ and taking solutions of Eq. (8) of the form

$$
\mathrm{H}_{0}=H_{0}(z) \exp \left(-i \alpha_{0} x\right)
$$

we obtain

$$
\frac{d^{2} H_{0}}{d z^{2}}-\frac{1}{\bar{\epsilon}_{1}} \frac{d \bar{\epsilon}_{1}}{d z} \frac{d H_{0}}{d z}+\left[\left(\bar{\epsilon}_{1}-\alpha_{0}^{2}\right)-\alpha_{0} \frac{\bar{\epsilon}_{1}}{\overline{\bar{\epsilon}}_{2}^{2}} \frac{d \bar{\epsilon}_{2}}{d z}\right] H_{0}=0
$$

The cutoff at $z=z_{c}$, where $\bar{\epsilon}_{1}=\bar{\epsilon}_{2}=0$ and $d \bar{\epsilon}_{1} / d z=d \bar{\epsilon}_{2} / d z \neq 0$, is a regular singular point. The behavior of the field near this point can be determined using the Method of Frobenius (Ince, 1956). Since the two solutions of the Indicial Equation are 0 and 2, in ascending powers of $\xi=z-z_{c}$, one solution of Eq. (20) is

$$
f_{1}(\xi)=\xi^{2}\left(1+c_{1} \xi+c_{2} \xi^{2}+\cdots\right.
$$

while a second solution may be cast in the form

$$
f_{2}(\xi)=K f_{1}(\xi) \ln \xi+1+\bar{d}_{1} \xi+d_{3} \xi^{3}+\cdots
$$

where a substitution into Eq. (20) gives

$$
K=0, d_{1}=-\alpha_{0} .
$$


It is interesting to note that in the case of an isotropic $(B=0)$ plasma, $K \neq 0$ when $\alpha_{0} \neq 0$, resulting in absolute values of the reflection coefficient lower than unity (Denisov, 1957). This phenomenon disappears for $\mathbf{B} \neq 0$.

For perturbations of the form

$$
\begin{aligned}
& \tilde{\epsilon}_{1}(x, z)=\widetilde{\varepsilon}_{1}(z) \exp (-i p x), \\
& \tilde{\epsilon}_{2}(x, z)=\widetilde{\varepsilon}_{2}(z) \exp (-i p x),
\end{aligned}
$$

and solutions of Eq. (9) of the form

$$
\mathrm{H}_{1}=H_{1}(z) \exp \left(-i \alpha_{1} x\right)
$$

where $\alpha_{1}=\alpha_{0}+p$, the equation for the scattered field becomes

$$
\begin{aligned}
\frac{d^{2} H_{1}}{d z^{2}} & -\frac{1}{\bar{\epsilon}_{1}} \frac{d \bar{\epsilon}_{1}}{d z} \frac{d H_{1}}{d z}+\left[\left(\bar{\epsilon}_{1}-\alpha_{1}^{2}\right)-\alpha_{1} \frac{\bar{\epsilon}_{1}}{\bar{\epsilon}_{2}^{2}} \frac{d \bar{\epsilon}_{2}}{d z}\right] H_{1} \\
& =\frac{d H_{0}}{d z} \frac{d}{d z}\left(\frac{\tilde{\varepsilon}_{1}}{\bar{\epsilon}_{1}}\right)-\widetilde{\varepsilon}_{1} H_{0}\left(1+\frac{\alpha_{0} p}{\bar{\epsilon}_{1}}\right) \\
& -\frac{\bar{\epsilon}_{1}}{\bar{\epsilon}_{2}}\left\{p \frac{d H_{0}}{d z} \frac{\widetilde{\varepsilon}_{2}}{\bar{\epsilon}_{2}}-\alpha_{0} H_{0}\left[\frac{d}{d z}\left(\frac{\widetilde{\varepsilon}_{2}}{\bar{\epsilon}_{2}}\right)+\frac{1}{\bar{\epsilon}_{2}} \frac{d \bar{\epsilon}_{2}}{d z}\left(\frac{\widetilde{\varepsilon}_{1}}{\bar{\epsilon}_{1}}-\frac{\widetilde{\varepsilon}_{2}}{\bar{\epsilon}_{2}}\right)\right]\right\} .
\end{aligned}
$$

As in the case for the ordinary mode, the solution of Eq. (24) may be cast in the form

$$
H_{1}(z)=\int_{-\infty}^{z} \frac{y_{1}(v) y_{2}(z)}{W(v)} S(v) d v+\int_{z}^{\infty} \frac{y_{1}(z) y_{2}(v)}{W(v)} S(v) d v
$$

where $S(z)$ is the r.h.s. of Eq. (24), and $y_{1}(z)$ and $y_{2}(z)$ are two indeperdent solutions of the corresponding homogeneous equation with Wronskian $W(z)=$ $W(0) \bar{\epsilon}_{1}(z)$. Similarly, in the vacuum region the scattered field is given by

$$
H_{1}(z)=\beta_{1}^{-1} \exp \left(i \beta_{1} z\right) \int_{0}^{\infty} \frac{A(v) S(v)}{\bar{\epsilon}_{1}(v)} d v
$$

where $\beta_{1}=\left(1-\alpha_{1}^{2}\right)^{1 / 2}$ and $A(z)$ is the solution of the homogeneous equation which in the vacuum region is of unit amplitude and in the evanescent region 
converges toward the subdominant WKB solution. The function $A(z)$ may readily be obtained from a numerical integration along the real $z$-axis with a small b'at finite value of $\nu$ (Freidberg et al., 1972). The integration must proceed from the evanescent region with initial conditions given by the WKB solution.

Finally, an integration by parts and use of Eq. (20) transform Eq. (26) into

$$
H_{1}(z)=\beta_{1}^{-1} \exp \left(i \beta_{1} z\right) \int_{0}^{\infty}\left[\widetilde{\varepsilon}_{1}(v) G_{1}(v)+\widetilde{\varepsilon}_{2}(v) G_{2}(v)\right] d v
$$

where

$$
\begin{aligned}
& G_{1}(v)=-\frac{1}{\bar{\epsilon}_{1}^{2}}\left[\alpha_{0} \alpha_{1} A(v) H_{0}(v)+\frac{d A(v)}{d v} \frac{d H_{0}(v)}{d v}\right], \\
& G_{2}(v)=-\frac{1}{\bar{\epsilon}_{2}^{2}}\left[\alpha_{1} A(v) \frac{d H_{0}(v)}{d v}+\alpha_{0} H_{0} \frac{d A(v)}{d v}\right] .
\end{aligned}
$$

Near the cutoff, where $\bar{\epsilon}_{1} \approx \bar{\epsilon}_{2}$ and $\widetilde{\varepsilon}_{1} \approx \widetilde{\varepsilon}_{2}$, the integrand of Eq. (27) may be approximated by the form

$$
-\frac{\widetilde{\varepsilon}_{1}}{\bar{\epsilon}_{1}^{2}}\left[\frac{d H_{0}(v)}{d v}+\alpha_{0} H_{0}(v)\right]\left[\frac{d A(v)}{d v}+\alpha_{1} A(v)\right]
$$

which is of the order of $\widetilde{\varepsilon}_{1}$ since by Eq. (23) the two terms in square brackets are of the order of $\bar{\epsilon}_{1}$. Thus Eq. (27) constitutes a well behaved (i.e., non-singular) solution of Eq. (24) in the vacuum region, $z<0$.

The flow of scattered energy across the plasma boundary at $z=0$ is

$$
S_{0}=\frac{c}{8 \pi}\left|\int_{0}^{\infty} \widetilde{\varepsilon}_{0}(v) G_{0}(v) d v\right|^{2}
$$

for the ordinary mode, and

$$
S_{1}=\frac{c}{8 \pi}\left|\int_{0}^{\infty}\left[\tilde{\varepsilon}_{1}(v) G_{1}(v)+\tilde{\varepsilon}_{2}(v) G_{2}(v)\right] d v\right|^{2}
$$

for the extraordinary mode. We now consider plasma irregularities of the form

$$
\widetilde{\varepsilon}_{i}(z)=g_{i}(z) \tilde{e}_{i}(z), \quad i=0,1,2
$$


expressed as the product of a slowly varying function $g_{i}(z)$ and a rapidly oscillating function $\tilde{e}_{i}(z)$. By assuming without loss of generality that $\tilde{e}_{i}(z)$ is spatially bounded and sufficiently smooth, we introduce the Fourier expansion

$$
\tilde{\varepsilon}_{i}(z)=g_{i}(z) \int_{-\infty}^{+\infty} \tilde{e}_{i}(q) \exp (-i q z) d q
$$

where $\tilde{e}_{i}(q)$ is taken as the Fourier transform of $\tilde{e}_{i}(z)$. By inserting Eq. (32) into Eqs. (18) and (27) we obtain

$$
\begin{aligned}
& E_{1}(z)=\beta_{1}^{-1} \exp \left(i \beta_{1} z\right) \int_{-\infty}^{+\infty} L_{0}(q) d q \\
& L_{0}(q)=\int_{0}^{+\infty} \tilde{e}_{0}(q) g_{0}(v) G_{0}(v) \exp (-i q v) d v
\end{aligned}
$$

for the ordinary wave, and

$$
\begin{aligned}
& H_{1}(z)=\beta_{1}^{-1} \exp \left(i \beta_{1} z\right) \int_{-\infty}^{+\infty} L_{1}(q) d q \\
& L_{1}(q)=\int_{0}^{+\infty}\left[\tilde{e}_{1}(q) g_{1}(v) G_{1}(v)+\tilde{e}_{2}(q) g_{2}(v) G_{2}(v)\right] \exp (-i q v) d v
\end{aligned}
$$

for the extraordinary wave. These expressions indicate that the major contribution to the scattered field originates from the Fourier components of $\tilde{e}_{i}(z)$ which match those of the product $g_{i}(z) G_{i}(z)$.

By inserting Eqs. (33) and (34) into Eqs. (29) and (30) we obtain

$$
S_{i}=\frac{c}{8 \pi}\left|\int_{-\infty}^{+\infty} L_{i}(q) d q\right|^{2}
$$

for the two modes of propagation.

For a random medium which satisfies the conditions

$$
<\tilde{e}_{i}(z)>=<\tilde{e}_{i}(q)>=0
$$

and

$$
<\widetilde{e}_{i}^{*}(q) \widetilde{e}_{j}\left(q^{\prime}\right)>=<\widetilde{e}_{i}^{*}(q) \tilde{e}_{j}(q)>\delta\left(q-q^{\prime}\right)
$$


where the angle brackets represent ensemble averages, we obtain

$$
<S_{i}>=\frac{c}{8 \pi} \int_{-\infty}^{+\infty}<\left|L_{i}(q)\right|^{2}>d q .
$$

This completes the set of equations describing the scattered field within the Born approximation.

\section{Numerical Examples}

In the following, we present some numerical results which illustrate the localization properties of fluctuation measurements using reflectometry in tokamaks. For the numerical simulation, we assume the electron density profile in our slab geometry to be similar to the electron density distribution (Fig. 1) on the equatorial plane of a typical TFTR tokamak discharge in the enhanced confinement regime (Strachan et al., 1987). Similarly, we assume the magnetic field profile is equal to the equatorial profile of the toroidal magnetic field in TFTR. Under these conditions, Fig. 2 indicates the variation of $\omega_{p}, \omega_{u c}=\left[\omega_{c}+\left(\omega_{c}^{2}+4 \omega_{p}^{2}\right)^{1 / 2}\right] / 2$, and $\omega_{u h}=\left(\omega_{p}^{2}+\omega_{c}^{2}\right)^{1 / 2}$. The first is the cutoff frequency for the ordinary mode and the other two are the upper cutoff and the upper hybrid resonance frequency of the extraordinary mode, respectively. Figure 2 shows that apart from a narrow region around the plasma boundary, the location of the upper cutoff for a given frequency is separated from that of the upper resonance by several free space wavelengths. This makes the upper hybrid resonance inaccessible to extraordinary waves propagating from the low-field side, as we have assumed in $\S 2$.

To investigate the localization properties of fluctuations measurements using microwave reflectometry in tokamaks, we take a density perturbation of the form

$$
\tilde{n}_{e}=\tilde{n} \exp \left[-\left(z-z_{c}\right)^{2} / \Delta^{2}\right] \cos \left[2 \pi\left(z-z_{c}\right) / \Lambda+\phi\right]
$$

corresponding to a Gaussian wave packet with width $\Delta$ and wavelength $\Lambda$. The scattered field in the vacuum region is obtained by inserting Eq. (37) into Eqs. 
(18) and (27). We shall consider two quite distinct cases in order to demonstrate the wave number dependence of the localization of measurement to the cutoff. In the first case, we choose $\Delta>\Lambda$. The normalized electric field $E_{1} / E_{0}$ at $z=0$ is shown in Figs. 3 and 4 fo: the two modes of propagation as a function of the wave packet center $z=z_{c}$ for $\tilde{n} / \bar{n}_{e}=1 \times 10^{-3}, \Delta=2 \mathrm{~cm}, \Lambda=0.5 \mathrm{~cm}$, and $\phi=\alpha_{0}=\alpha_{1}=0$. The wave frequencies are $6 \times 10^{10} \mathrm{~Hz}$ for the ordinary mode (Fig. 3) and $1.4 \times 10^{11} \mathrm{EIz}$ for the extraordinary mode (Fig. 4). For these wave frequencies the two modes of propagation have the same cutoff location, however, the central location of the plasma wave packet $z_{c}$ which maximizes the scattered field is quite aifferent in the two cases. In fact, for the extraordinary mode the scattered field is strongly weighted tcwards density fluctuation near the cutoff, while for the ordinary mode the scattered field is largest when the density perturbation is located woll away from the cutoff. Since the scattered field origunates from a region where the spatial variation of the lcwest order wave pattern matches that of the density perturbation, the major difference between the two cases is in the different ratios of $\Lambda / \lambda_{0}$. For $\Lambda \leq \lambda_{0}$, the matching occurs away from the turning point, while for $\Lambda \gg \lambda_{0}$ matching occurs only near the turning point. As a rule, the latter case requires that $\Lambda \geq 2.5 \lambda_{0}$. Thus, assuming a power la $w$ dependence for plasma fluctuations at high wavenumbers, the extraordinary mode should dramatically decrease the contribution to the scattered power from fluctuations away from the cutoff.

In the second case, we choose $\Delta \leq \Lambda$. The normalized electric field $E_{1} / E_{0}$ at $z=0$ is shown in Figs. 5 and 6 as a function of $z_{c}$ for the extraordinary mode with a frequency of $1.4 \times 10^{11} \mathrm{~Hz}$ and for $\Delta=2 \mathrm{~cm}, \Lambda=10$, and $\alpha_{0}=\alpha_{1}=0$. The results of Fig. 5 were obtained with $\phi=0$ (symmetric perturbation) while those of Fig. 6 were obtained with $\phi=-\pi / 2$ (antisymmetric perturbation). In both cases, the value of $\bar{n}$ in Eq. (37) was adjusted such that $\max \left(\tilde{n}_{e}\right)=1 \times 10^{-3} \bar{n}_{e}$. Although in both cases the scattered field is largest when the plasma perturbation is near the cutoff, the antisymmetric case ( $F \mathrm{~g}$. 6) shows greater relative locailization near the cutorf than for the symmetric caste 
(Fig. 5). This result is readily understood in the geometric optics approximation where the phase of the scattered field is proportional to a line integral through the perturbation up to the reflecting layer. Moreover, away from the cutoff, the phase perturbation of the refiected wave varies like $k_{0}^{-1} \widetilde{n}_{e}$, while at the cutofi, for a given displacement of the reflecting layer $\delta r$, the phase varies like $k_{0} \delta r$. Thus, the relative contribution to the total phase variation from the cutoff region sicales like $k_{0}^{2}$ so that the extraordinary mode has superior localization properties even for large sizr plasma fluctuations.

\section{Conclusion}

In conclusion, we have derived within the Born approximation the equations for the electromagnetic field scattered by density fluctuations in an inhomogeneous magnetized plasma in the presence of a cutoff. Numerical results for both the ordinary and the extraordinary mode show that the scattered field is strongly enhanced near the turning point when the spatial scale length of density fluctuations is greater than the free space wavelength of the probing beam. These results indicate that microwave reflectometry may be used for obtaining localized information on the distribution of fine scale density fluctuations in the range $k_{\perp} \rho_{i} \ll 1$ in tokamaks, provided $k_{\perp}<<k_{0}$. The latter condition suggests the use of the extraordinary mode since it requires higher wave frequencies than the ordinary mode.

The results however are qualified by the nature of our approximations, where a linearized treatment of the scattered field is used. Extension of this analysis to strong scattering from plasma fluctuation requires an alternative approach, however the localization of the scattered field to the cutoff for long wavelength fluctuations is expected to hold in general.

\section{Acknowledgments}

This work was supported by U.S. Department of Energy Contract No. DEAC02-76-CHO-3073. 


\section{References}

Budden K. G. (1961) Radio Waves in the Ionosphere. Cambridge University Press, London.

Cripwell P., Costley A. E., and Hubbard A. E. (1989) Proc. of $16^{\text {th }}$ Europ. Conf. on Contr. Fusion and Plasma Phys., Venice, Italy, Vol. 13B, 75.

Denisov N. G. (1957) Soviet Phys. JETP 4, 544.

Doane J., Mazzucato E., and Schmidt G. (1980) Rev. Sci. Instrum. 52, 12.

Freidberg J. P., Mitchell R. W., Morse R. L. and Rudsinski L. I. (1972) Phys. Rev. Lett. 28, 795.

Ginzburg V. L. (1964) Propagation of Electromagnetic Waves in Plasmas. Pergamon Press, New York.

Haas F. A. and Thyagaraja (1986) Physics Reports 143, 240.

Heading J. (1962) An Introduction to Phase Integral Methods. Methuen, London.

Horton W. (1984) Handbook of Plasma Physics Vol. 2. North Holland, Amsterdam.

Ince E. L. (1927) Ordinary Differential Equations. Longmans Green, London.

Jeffreys H. and Jeffreys B. S. (1972) Method of Mathematical Physics. Cambridge University Press, London.

Kadomtsev B. B. and Pogutse O. P. (1970) Reviews of Plasma Physics Vol. 5. Consultants Bureau, New York.

Liewer P. (1985) Nucl. Fusion 25, 543.

Mazzucato E. (1975) Density Fluctuations in the Adiabatic Toroidal Compressor. Princeton University Plasma Physics Lab. Rep. MATT-1151. 
Mazzucato E. (1976) Phys. Rev. Lett. 36, 792.

Pitteway M. L. (1958) Proc. Roy. Soc. A 252, 556.

Strachan J. D., Bitter M., Ramsey A. T., Zarnstorff M. C., Arunasalam V., Bell M. G., Bretz N. L., Budny R., Bush C. E., Davis S. L., Dylla H. F., Efthimion P. C., Fonck R. G., Fredrickson E., Furth H. P., Goldston R. G., Grisham L. R., Grek B., Hawryluk R. J., Heidbrink W. W., Hendel H. W., Hill K. W., Hsuan H., Jaehnig K. P., Jassby D. L., Jobes F., Johnson D. W., Johnson L. C., Kaita R., Kamperschroer J., Knize R. Z., Kozub T., Kugel H., LeBlanc B., Levinton F., La Marche P. H., Manos D. M., Mansfield D. K., McGuire K., McNeill D. H., Meade D. M., Medley S. S., Morris W., Mueller D., Nieschmidt E. B., Owens D. K., Park H., Schivell J., Schilling G., Schmidt G. L., Scott S. D., Sesnic S., Sinnis J. C., Stauffer F. J., Stratton B. C., Tait G. D., Taylor G., Towner H. H., Ulrickson M., von Goeler S., Wieland R., Williams M. D., Wong K-L., Yoshikawa S., Young K. M., and Zweben S. J. (1987) Phys. Rev. Lett. 58, 1104.

Surko C. M. and Slusher R. E. (1976) Phys. Rev. Lett. 37, 1747.

Tang W. M. (1978) Nucl. Fusion 18, 1089. 


\section{Figure Captions}

Fig. 1. Electron density distribution on the equatorial plane for a TFTR discharge.

Fig. 2. Profile of upper cutoff $f_{u c}$ frequency (solid line), upper hybrid frequency $f_{u h}$ (dot line) and plasma frequency $f_{p}$ (dash line) for a TFTR discharge with the derisity profile of Fig. 1 and a magnetic field of $50 \mathrm{kG}$.

Fig. 3. Normalized scattered field $E_{1} / E_{0}$ (solid line) at $z=0$ for the ordinary mode as a function of the position of a density wave packet which is shown by the dashed line centered at the cutoff point; $\tilde{n} / \bar{n}_{e}=1 \times 10^{-3}, \omega / 2 \pi=60 \mathrm{GHz}$, $\alpha_{0}=\alpha_{1}=0$.

Fig. 4. Same as for Fig. 3 for the extraordinary mode with $\omega / 2 \pi=140 \mathrm{GHz}$ and $\alpha_{0}=\alpha_{1}=0$.

Fig. 5. Normalized scattered field $E_{1} / E_{0}$ (solid line) at $z=0$ for the extraordinary mode as a function of the position of a symmetric density perturbation which is shown by the dashed line centered at the cutoff point; $\tilde{n} / \bar{n}_{e}=1 \times 10^{-3}$, $\omega / 2 \pi=140 \mathrm{GHz}$ and $\alpha_{0}=\alpha_{1}=0$.

Fig. 6 Same as for Fig. 5 for an antisymmetric density perturbation. 


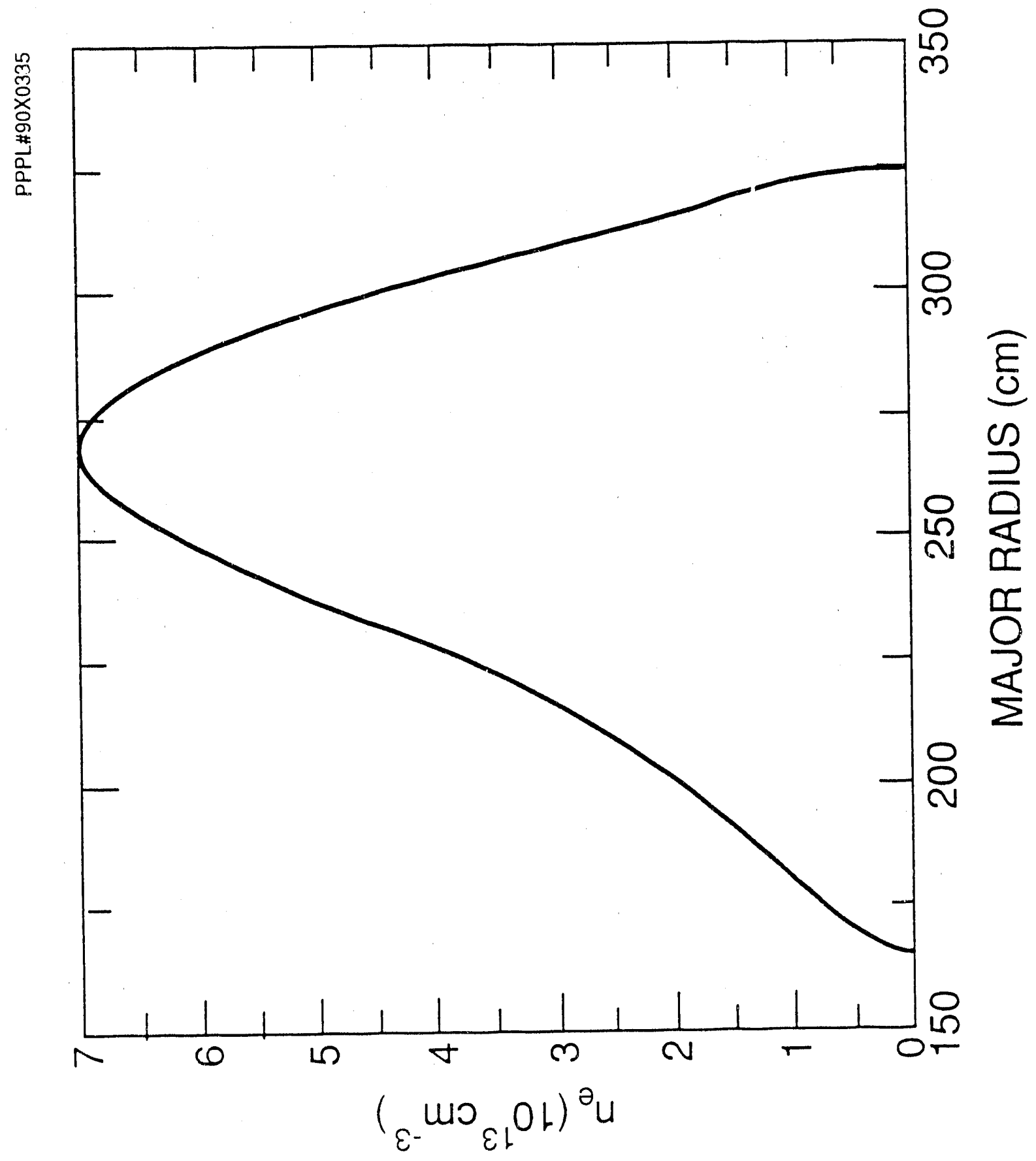




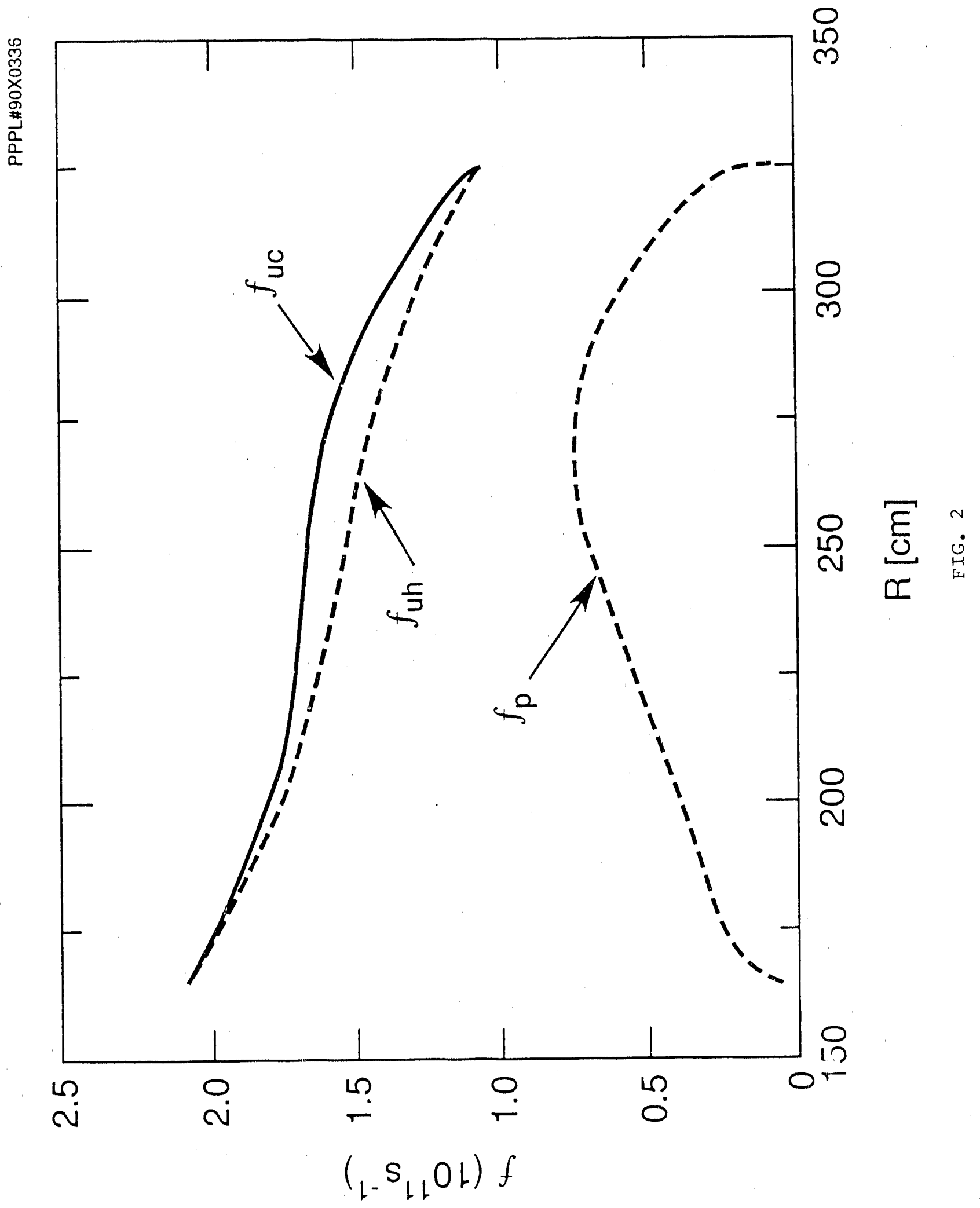




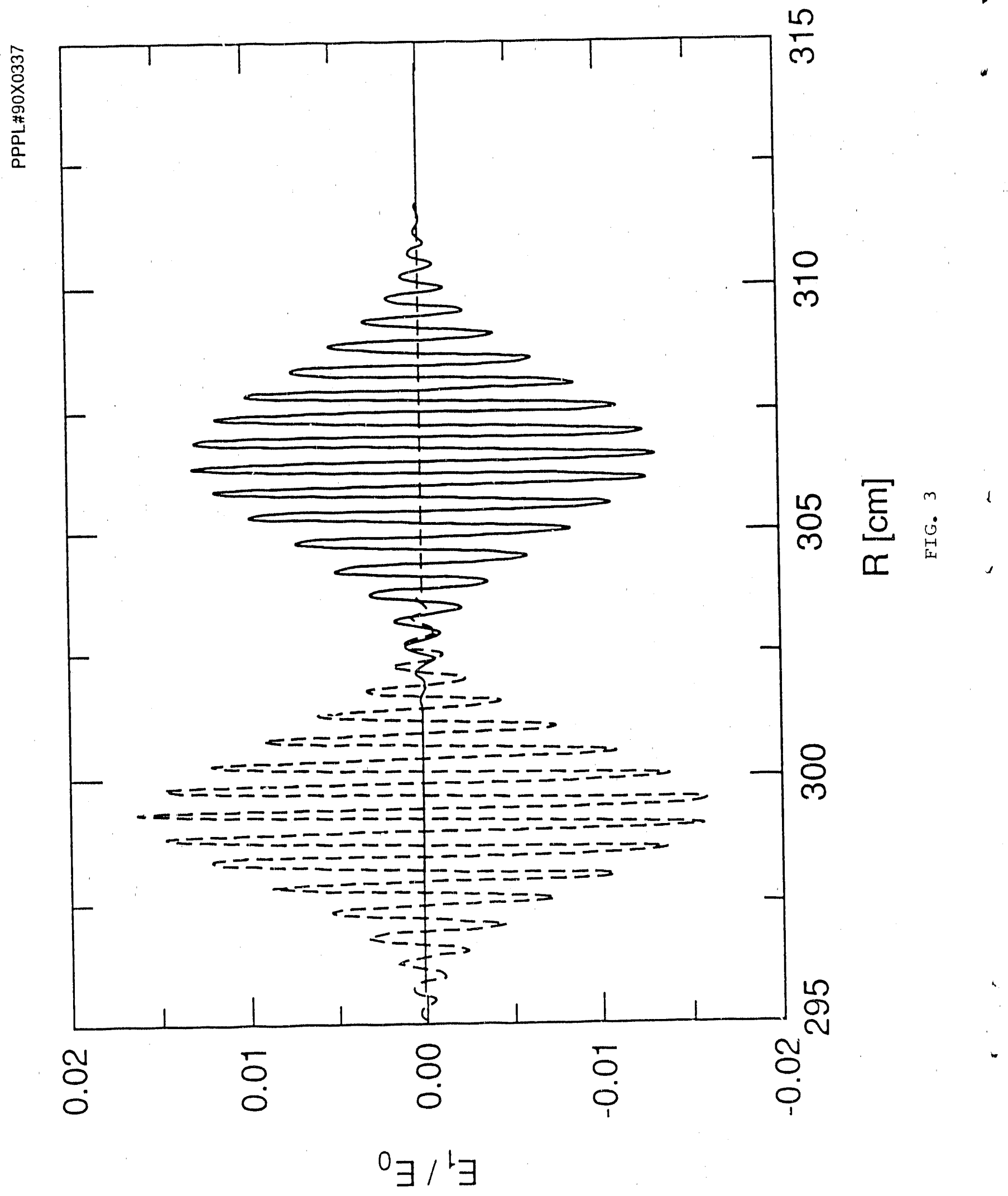




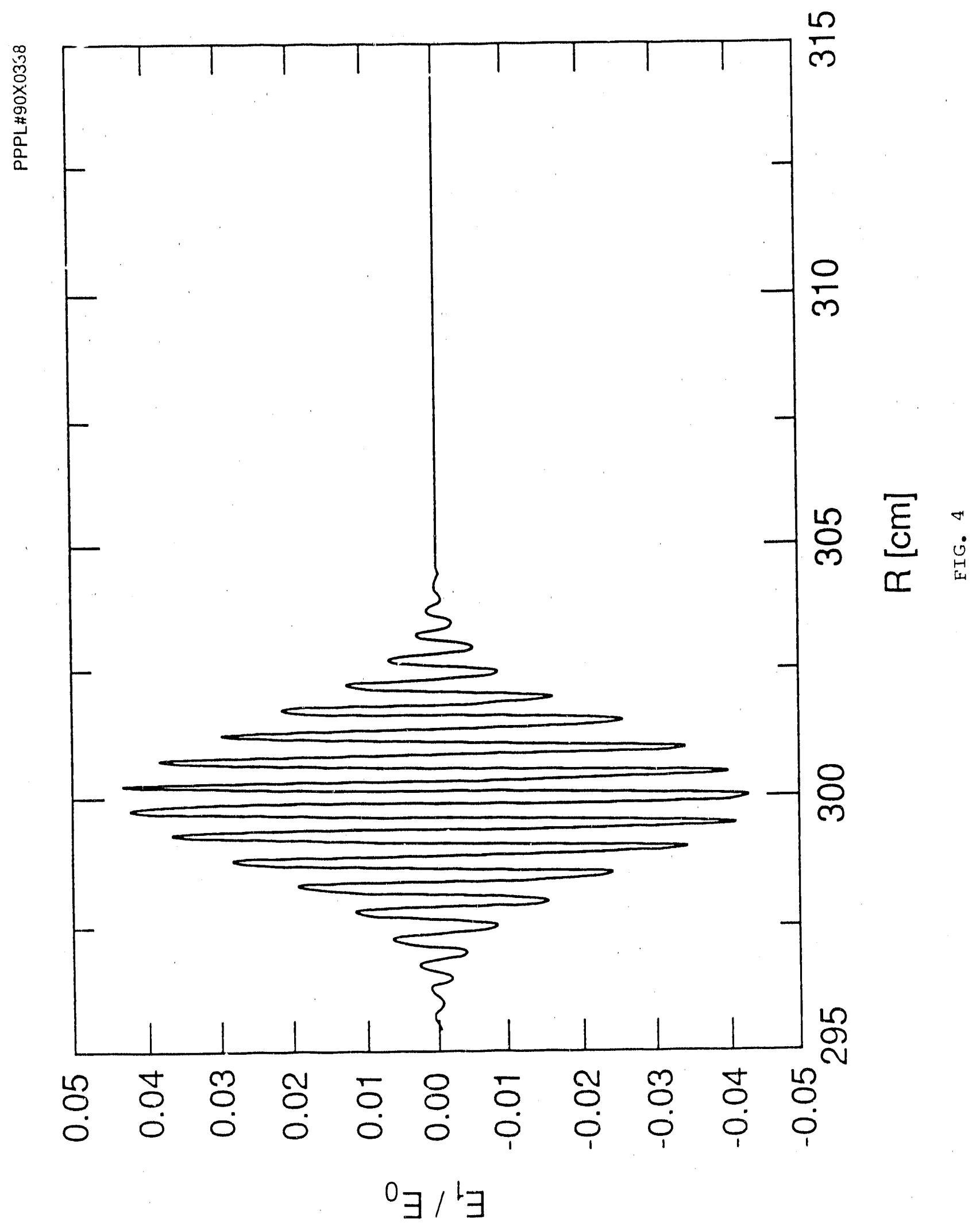




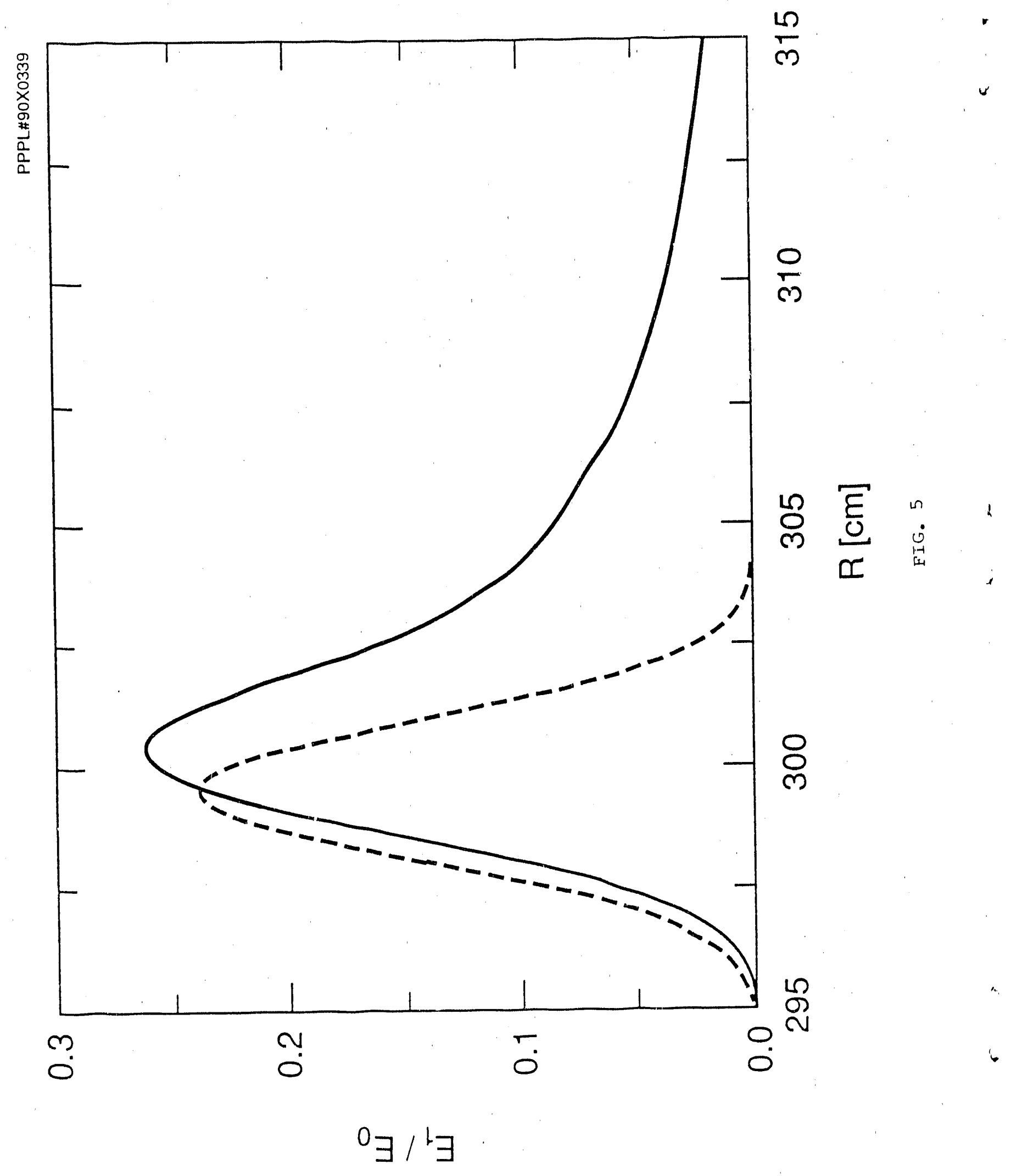




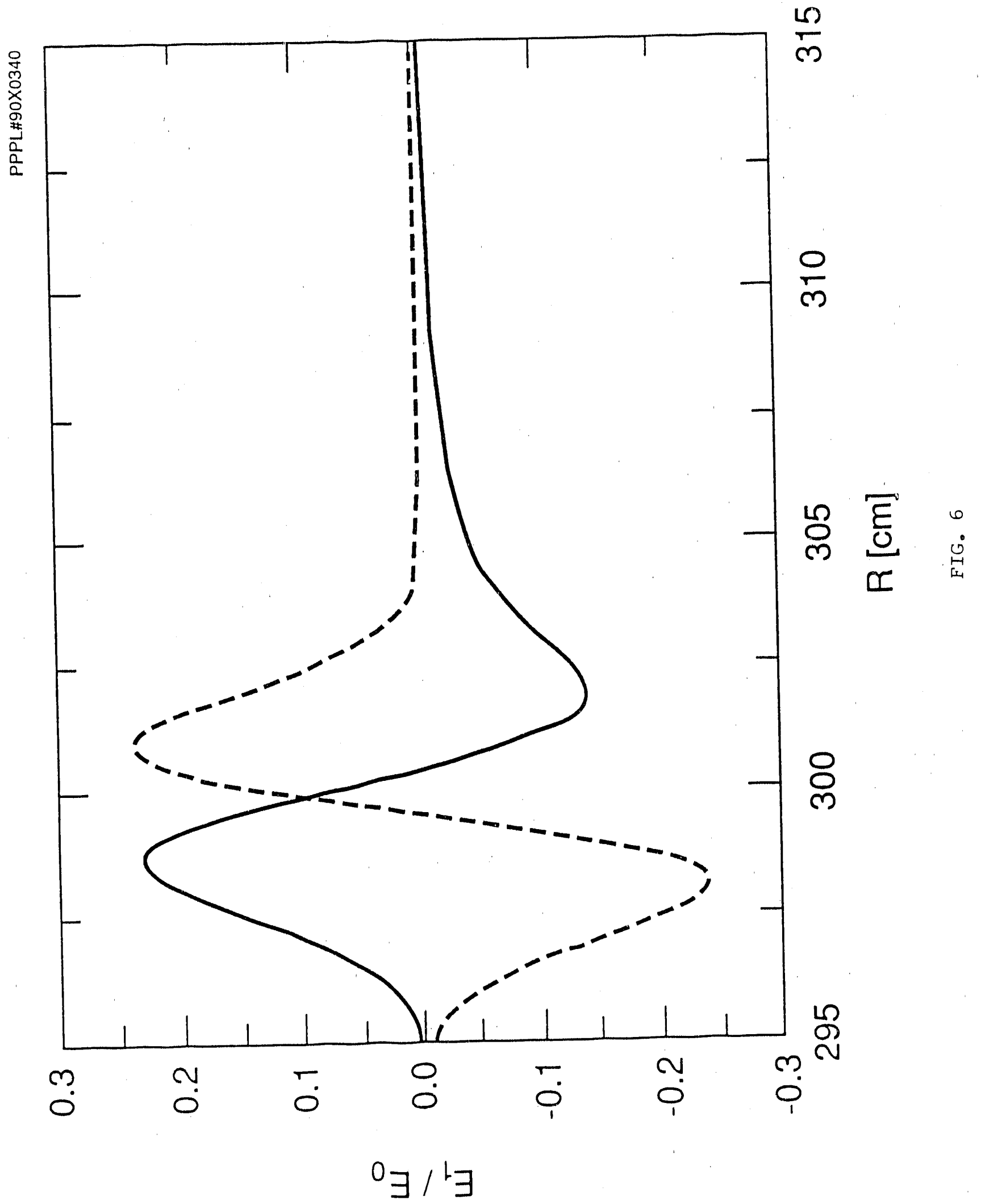

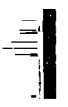



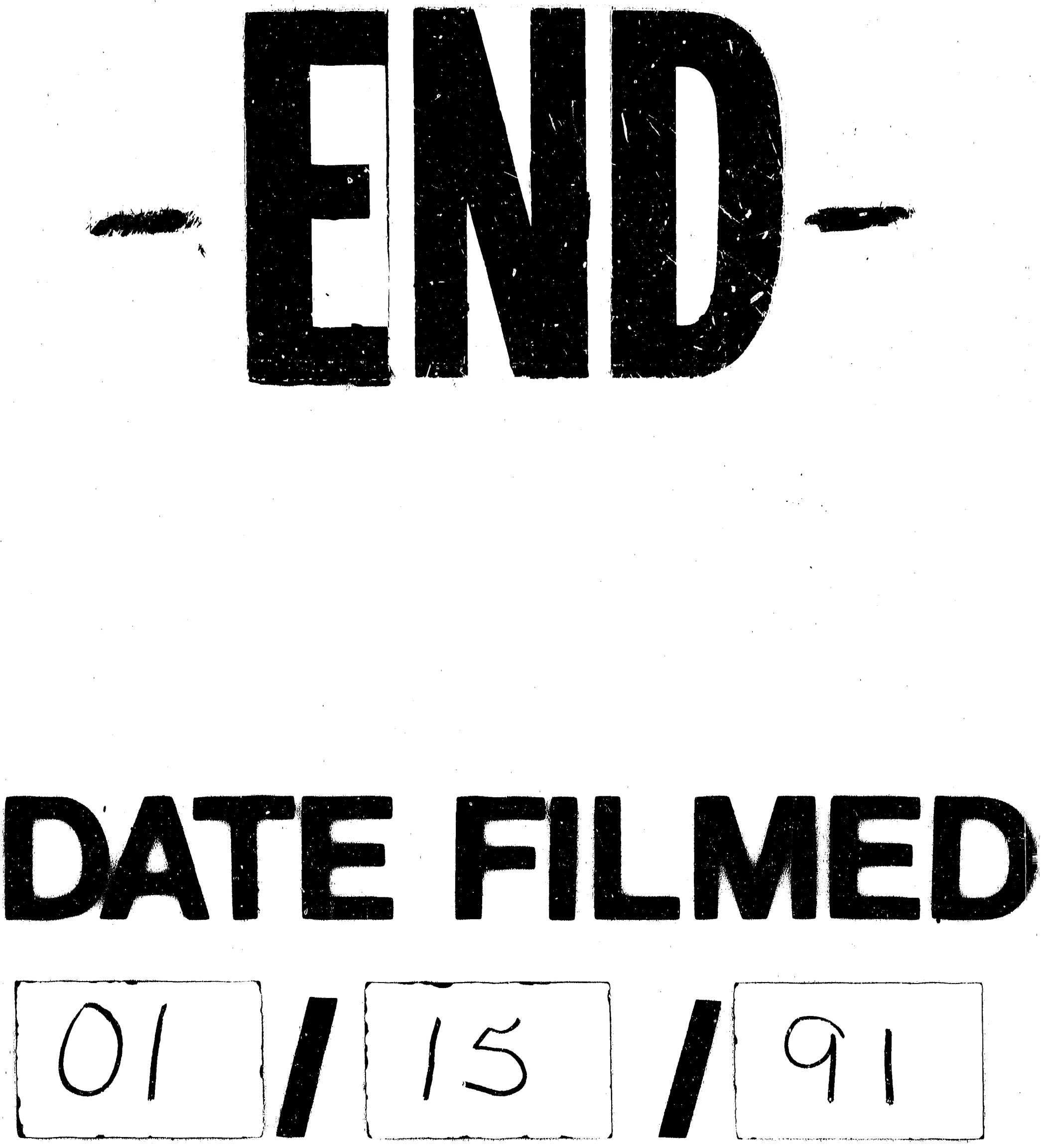
